\title{
Análisis de vulnerabilidad ambiental de la cuenca alta del río San Pedro, estado Miranda, Venezuela
}

\author{
Environmental vulnerability analysis of high river basin san Pedro, Miranda, Venezuela
}

\author{
${ }^{\bullet}$ Haideé Coromoto Marín' ${ }^{1}$, Carlos Alfredo Bravo Medina ${ }^{1}$, Nelly Narcisa Manjarrez Fuentes ${ }^{1,2}$, Emma Torres Navarrete ${ }^{3}$ \\ ${ }^{1}$ Universidad Estatal Amazónica (UEA), Departamento de Ciencias de la Tierra. Km 1 vía a Tena. CP 160150. \\ Puyo-Pastaza.`hmarin@uea.edu.ec; cbravo@uea.edu.ec \\ ${ }^{2}$ Universidad Técnica Estatal de Quevedo. Unidad de Estudios a Distancia. Campus Manuel Haz Álvarez. \\ Av. Quito Km 1.5 vía Santo Domingo de los Tsáchilas.EC.120501.Quevedo,Ecuador.nmanjarrez@uteq.edu.ec \\ ${ }^{3}$ Universidad Técnica Estatal de Quevedo. Facultad de Ciencias Pecuarias. Carrera de Ingeniería Zootécnica. Campus Finca \\ Experimental “La María”. CP. 121250. Km. 7 1⁄2 vía El Empalme, cantón Mocache. Los Ríos. Ecuador. etorres@uteq.edu.ec
}

Rec.: 20.03.2017. Acept.: 10.07.2017 Publicado el 1 de diciembre de 2017

\section{Resumen}

$\mathrm{S}_{\mathrm{y}}^{\mathrm{e}}$ caracterizó la vulnerabilidad ambiental por inundación desplazamiento en comunidades asentadas en la Cuenca alta del río San Pedro, municipio Guaicaipuro, estado Miranda; Venezuela usando SIG. El área de estudio se dividió en dos zonas, una ligeramente intervenida (A) y otra fuertemente intervenida (B), para obtener los mapas temáticos usados como indicadores, los cuales se estandarizaron mediante la técnica de re-clasificación asignándoles valores numéricos en una escala del 1 al 5. Los resultados muestran que la vulnerabilidad por inundación en la subcuenca siguió la siguiente tendencia: clase media (3) $33.75 \%>$ clase baja (2) $31.91 \%>$ clase muy baja (1) $26.86 \%>$ clase alta (4) $6.12 \%>$ clase muy alta (5) $1.37 \%$, no obstante las clases alta y muy alta se corresponde con comunidades cercanas al río, caracterizadas por la presencia de desarrollos urbanos, alta actividad agrícola, sugiriendo un alto riesgo por inundación. La distribución espacial de la vulnerabilidad por desplazamiento sugiere que predomina la clase media (3) y baja (2), las cuales representan el 50.58 y $36.25 \%$ respectivamente, mientras que el resto de las clase como alta (4), muy baja (1) y muy alta (5) mostraron porcentajes de $9.37,3.29$ y 0.41 respectivamente. Las clases de vulnerabilidad alta y muy alta están localizadas en el área de mayor intervención (Zona B), con un patrón muy irregular asociado a suelos con altas pendientes $(>30 \%)$ y manejo de suelo con cultivos pocos protectores como las hortalizas, favoreciendo procesos de degradación e incidiendo en la sustentabilidad de la zona. Los resultados obtenidos han servido para discriminar cuales comunidades son más vulnerables y establecer prioridades en cuanto a la intervención para minimizar los impactos ambientales.

Palabras clave: Vulnerabilidad ambiental, SIG, manejo intensivo, sustentabilidad.
Q nvironmental vulnerability by flooding and displacement Cof the communities located in the upper San Pedro River, Guaicaipuro, Miranda state, Venezuela was characterized using GIS. The area was divided into two parts: a slightly intervened (A) and strongly intervened (B) to obtain thematic maps used as indicators, which were standardized by re-classification technique, assigned numerical values on a scale of 1 to 5 . The results show that the flood vulnerability in the sub watershed showed the following trend: middle class (3) $33.75 \%>$ class 2 (low) $31.91 \%>$ class 1 (very low) 26.86\%> Class 4 (high) $6.12 \%>$ class 5 (very high) $1.37 \%$, despite the high and very high classes correspond to communities near the river (San Pedro, Rio Arriba and Andres Bello), characterized by the presence of urban development, high agricultural activity, which suggests a high flood risk. The spatial distribution of displacement vulnerability suggests that predominates middle (3) and lower (2), which represents 50.58 and $36.25 \%$ respectively, while the rest of the upper class (4), very low (1) to very high (5) showed percentages of $9.37,3.29$ and 0.41 respectively. The classes of high and very high vulnerability are located in the area of major intervention (Zone B), with a very irregular pattern associated with steep slopes (>30\%) and soils with agricultural managements systems with few protectors crops like vegetables, where land preparation leave uncovered soil surface, favoring degradation processes and affecting the sustainability of the area. The results obtained have served to discriminate which communities are most vulnerable and establish priorities in terms of intervention to minimize environmental impacts.

Key words: Environmental vulnerability, GIS, intensive management, sustainability. 


\section{Introducción}

$\mathrm{L}$ as cuencas hidrográficas son ecosistemas de gran importancia ambiental por su gran diversidad biológica, paisajística, cultural y prioritariamente por su capacidad de retención y regulación de agua (Smith y Olsen, 2010). Además ofrecen una oportunidad para efectuar cambios transformadores a gran escala que promueven el bienestar humano y protegen los ecosistemas que sustentan la vida en un futuro cercano (Esmail y Geneletti, 2017). En base a ello, comprender los procesos e interrelaciones que ocurren en una cuenca hacen necesario la caracterización de los recursos y de las condiciones biofísicas, socioeconómicas y ambientales (Marín y Bravo, 2013). Los factores del riesgo derivan de la relación dinámica entre las llamadas amenazas físicas y las vulnerabilidades de una sociedad o un componente en particular de la misma. Las amenazas son eventos físicos latentes, o sea probabilidades de ocurrencia de eventos físicos dañinos en el futuro, y pueden clasificarse genéricamente de acuerdo con su origen, como "naturales", "socio-naturales", o "antropogénicos" (Lavell, 2003). La vulnerabilidad puede entenderse como la posibilidad o inminencia de pérdida de biodiversidad, recursos (suelo y aguas) o productividad de un agroecosistema o de sus características socio-culturales claves frente a un proceso amenazante ocurrente o inminente (Altieri y Nicholls, 2013). También la vulnerabilidad está asociada con el grado de susceptibilidad o de incapacidad de un sistema para afrontar los efectos adversos del cambio climático y en particular la variabilidad del clima y los fenómenos extremos. La vulnerabilidad se puede evaluar en tres dimensiones (exposición, intensidad e impacto) y dependerá del carácter, magnitud y rapidez del cambio climático a que esté expuesto un sistema, y de su sensibilidad y capacidad de adaptación (IPCC, 2008; Smith y Olensen, 2010). El análisis de los factores de riesgo para la zona de la Cuenca alta del río San Pedro, indican que las amenazas derivan en una intersección de actividades antrópicas como por ejemplo deforestación, desestabilización de laderas por avance de la frontera agrícola, siembra de hortalizas con manejo convencional (monocultivo) y el asentamiento de comunidades en ambientes frágiles (Marín y Bravo, 2013). Así, un número importante de eventos físicos como inundaciones, deslizamientos, sequías, erosión de suelos y colapsos de tierra son generados o acentuados por dichas prácticas. Con base a ello, la evaluación de la vulnerabilidad en esta zona constituye un proceso prioritario mediante el cual se determina el grado de susceptibilidad y predisposición al daño o pérdida de un elemento o grupo de elementos económicos, sociales y humanos expuestos ante una amenaza particular, y los factores y contextos que pueden impedir o dificultar de manera importante la recuperación, rehabilitación y reconstrucción con los recursos disponibles en la unidad social afectada (Wilchex, 1993; Buch y Turcios, 2003; González et al., 2003). El Objetivo de este trabajo fue establecer niveles de vulnerabilidad ambiental de las comunidades localizadas en la Cuenca Alta del río San Pedro, Miranda, Venezuela; basados en la selección de algunos parámetros que, por un lado sirvan de indicadores, pero por otro aporten información importante como herramienta de planificación para el manejo sustentable.

\section{Materiales y métodos}

$\mathrm{P}_{\mathrm{A}}^{\mathrm{a}}$ ra este estudio se seleccionó el tramo de la Subcuenca Alta del río San Pedro (CARSP), ubicada dentro el Parque Nacional Macarao, el cual se encuentra ubicada dentro de la Serranía Litoral de la Cordillera de la Costa Central al suroeste de Caracas. Dicho parque comprende una superficie de aproximadamente 15000 ha, abarcando las cuencas de los ríos Macarao, San Pedro y Jarillo o Lagunetas, importantes fuentes de agua para Caracas y otras ciudades del norte de Venezuela (Castillo y Salas, 2006).

Predomina principalmente un paisaje montañoso de pendientes medias entre 26 y $51 \%$, con una altitud que varía entre los $1000 \mathrm{msnm}$, en las partes más bajas ubicadas al fondo del valle, hasta los 2098 msnm en el Alto de León, el punto más elevado localizado en el lindero norte. Sus suelos son moderadamente susceptibles a la erosión (Veitia y Malapina, 1994; Díaz, 2002). La zona presenta una precipitación anual que oscila entre 1000 y $1400 \mathrm{~mm}$ con una época de sequía que se extiende desde noviembre hasta abril, con lluvias periódicas en los meses restantes. La temperatura promedio anual fluctúa entre los $16^{\circ} \mathrm{C}$ y los $26^{\circ} \mathrm{C}$ (Castillo y Salas, 2006).

La vegetación está compuesta principalmente por sabanas, bosques semi-deciduos y bosques montanos siempre verdes, que en los puntos más altos llegan a alcanzar alturas de 25 a $30 \mathrm{~m}$, con dos o tres estratos arbóreos, abundancia de epífitas y un sotobosque relativamente denso (Castillo y Salas, 2006). Dentro del parque también hay especies exóticas que fueron plantadas con la finalidad de recuperar áreas que habían sido degradadas por el uso agrícola.

Las especies de árboles más comunes son el cedro (Cedrela odorata), el samán (Albisia saman), el palo maría (Triplaris caracasana), el bucare (Erythrina poeppigiana), el araguaney (Tabebuia chrysantha), el pardillo (Cordia alliodora), la majagua (Heliocarpus americanus) y el quiripití (Clusia minor). La palma prapa (Wettinia praemorsa) y la palma de cera o palma bendita (Ceroxylon interruptum) son comunes, así como también las epífitas de las familias de las bromelias (Tillandsia sp.) y de las orquídeas (Epidendrum sp.). En las sabanas predomina la gramínea capín melao (Melinis minutiflora) y una hierba desconocida perteneciente a la familia de las compuestas o asteraceae (Veitia y Malapina, 1994). Las plantaciones de especies exóticas están conformadas principalmente por pino ciprés (Cupressus lusitanica), casuarina o pino australiano (Casuarina 
equisetifolia) y eucalipto (Eucalyptus robusta) (Castillo y Salas, 2006).

El parque alberga 263 especies de este grupo, de las cuales 12 son endémicas de Venezuela. Entre ellas el tapaculo (Scytalopus caracae), la granicera hermosa (Pipreola formosa), el tucancito esmeralda (Aulacorhynchus sulcatus) y el colibrí pechiazul (Sternoclyta cyanopectus) (Castillo y Salas, 2006). También se encuentran algunas especies consideradas amenazadas a nivel internacional tales como el paují copete de piedra (Crax pauxi), el atrapamoscas cerdoso venezolano (Phylloscartes venezuelanus), y la perdiz montañera (Odontophorus columbianus) (IUCN, 2004).

La Subcuenca Alta del río San Pedro (CARSP), abarcó una superficie de $30.8 \mathrm{~km}^{2}$, lo que representa el $24.44 \%$ de la superficie total de la Cuenca con $126 \mathrm{~km}^{2}$, localizada entre las coordenadas geográficas $10^{\circ} 20^{\prime} 00^{\prime \prime}$ y $10^{\circ} 25^{\prime} 00^{\prime \prime}$ de Latitud Norte y $67^{\circ} 03^{\prime} 00^{\prime \prime}$ y $67^{\circ} 10^{\prime} 00^{\prime \prime}$ de Longitud Oeste. Esta zona está ubicada en la región de los altos Mirandinos formando parte de la cordillera de la costa, parroquia San Pedro, municipio Guaicaipuro, estado Miranda en la parte norte de Venezuela (Figura 1).

El área de estudio se dividió en dos zonas, una no intervenida (A) y otra intervenida (B) (Figura 2). La metodología propuesta para medir la vulnerabilidad ambiental de las comunidades por inundación y por deslizamiento se consideró los siguientes parámetros: a) pendiente, b) distancia de las comunidades al río, c) intensidad en el uso de la tierra y d) elevación.

Para el manejo de la información proveniente de la base de datos se utilizó el software libre gvSIG versión 1.12, lo cual permitió la construcción de las diferentes capas vectoriales y de imágenes para elaborar los distintos mapas temáticos. previamente, se obtuvo el modelo digital de elevación (MDE) interpolando las curvas de nivel (Campo de elevación) distanciadas cada 25 metros a partir de una capa vectorial, el cual fue rasterizado y convertido en imagen.

Se establecieron cinco rangos de altitud o elevación (msnm) considerando las características morfológicas de la subcuenca: 1040-1222, 1222-1404, 1404-1586, 1586-1768 y 1768-1950. A partir del mapa del MDE se procedió al cálculo o análisis de relieve usando el método de Ajuste a Polinomio de Grado 2 propuesto por Zevenbergen \& Thorne (1987), lo que permitió la obtención del mapa de pendiente con rangos de: $0-15,15-30,30-45,45-60$ y $>60$. La red de drenaje fue delineada a partir del MDE, utilizando los valores de acumulación de flujo, que indica la cantidad de celda aguas arribas y vierten sobre una celda dada. Una vez obtenida la hidrografía de la Cuenca se establecieron distintos rangos "buffers" de 0-25, 25-50, 50-75, 75-100 y > $100 \mathrm{~m}$, sobre la base de las características morfométricas del río San Pedro y la distancia de las viviendas y de las unidades agrícolas.

El uso de la tierra se obtuvo a partir de una imagen satelital Fusionada Spot con un tamaño de pixel de $2.5 \mathrm{~m}$ con las tres bandas espectrales (verde, azul y rojo), y fue procesada mediante una clasificación no supervisada a través del algoritmo isodata (Chuvieco, 2008), que agruparon cinco clases: bosque denso, bosque bajo, vegetación baja/matorrales, suelo desnudo/cultivo de porte bajo manejado de manera convencional; zona residencial rural (vivienda, galpones).

El procesamiento geoespacial del modelo cartográfico se basó en la asignación de códigos numéricos enteros a las categorías a través de la técnica de la re-clasificación de cada una de las capas temáticas o indicadores en una escala del 1-5, las cuales se conjugaron a fin de obtener un único valor luego

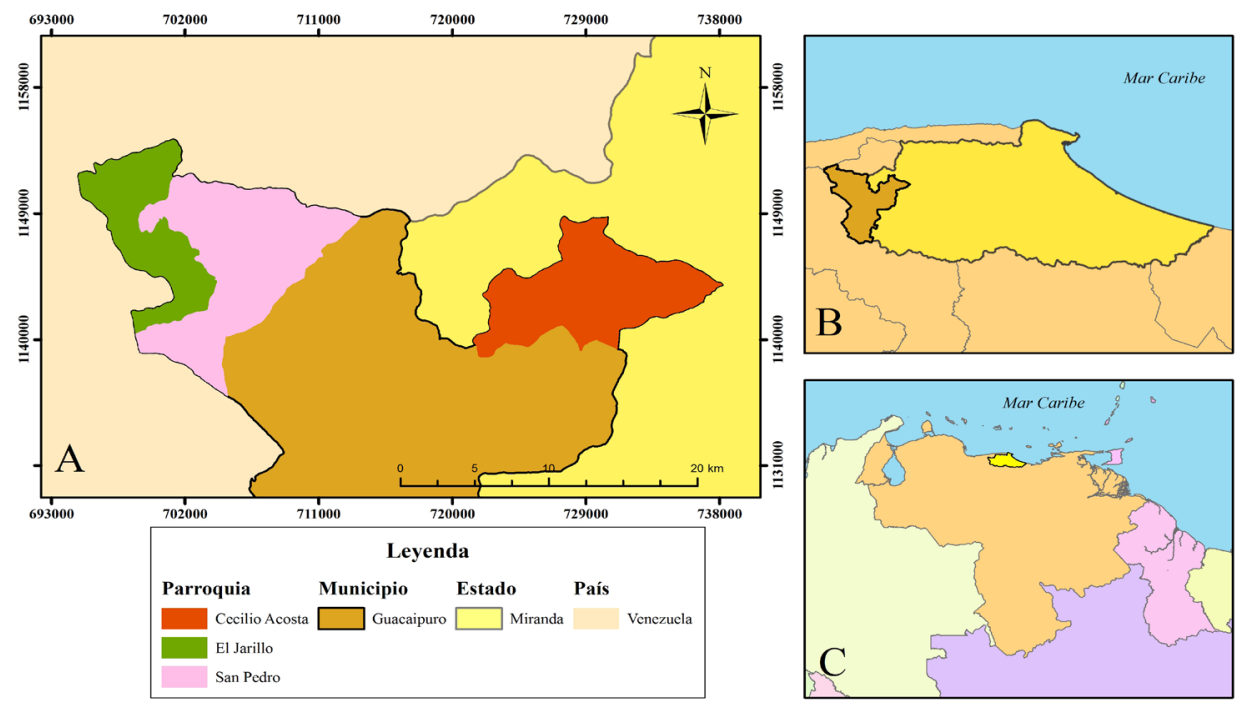

Figura 1. (A) Zonas de estudio en la Cuenca Alta del río San Pedro (CARSP) y (B) su ubicación relativa con el estado Miranda y (C) Venezuela. 


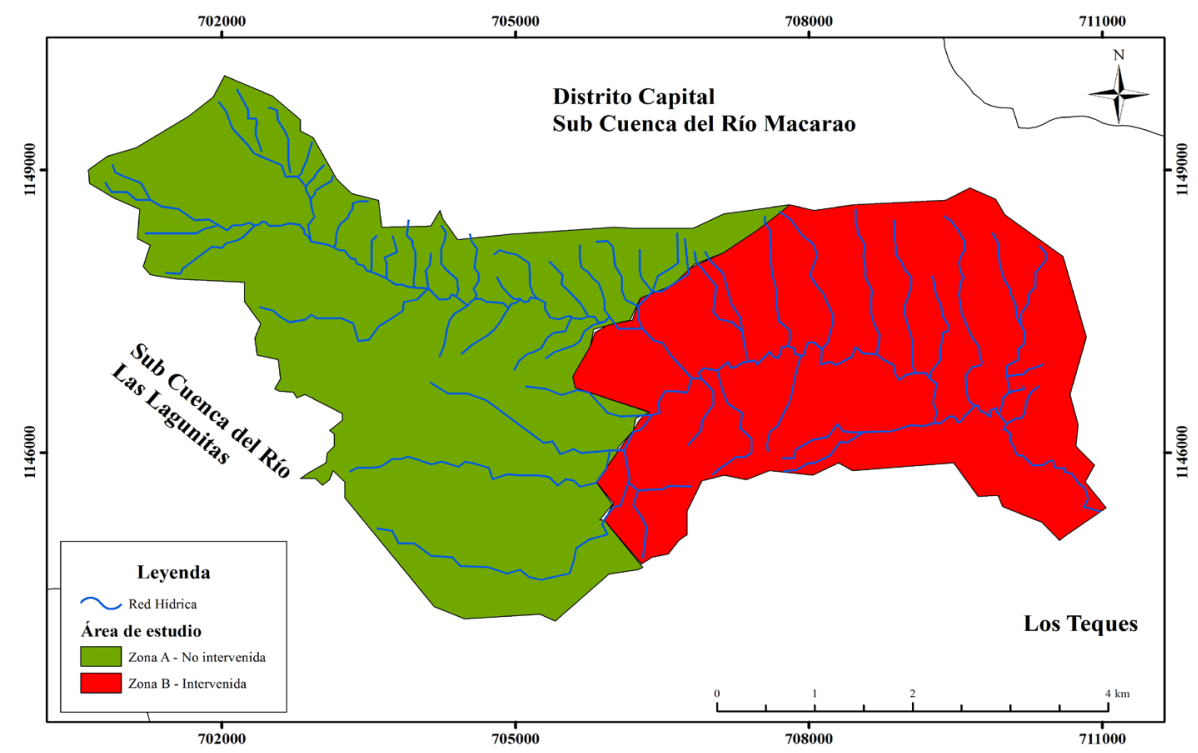

Figura 2. Zonas de Estudio en la Cuenca Alta del río San Pedro, estado Miranda, Venezuela

de la combinación de las mismas. Posteriormente se sumaron los distintos mapas reclasificados para obtener los mapas de vulnerabilidad a una escala cuyos rangos oscilaron de 3 a 15 . Obtenidos estos mapas, se aplicó una nueva reclasificación con escala de 1 a 5 que permitió la categorización final de los niveles de vulnerabilidad ambiental en cinco clases: muy baja (1), baja (2), media (3), alta (4) y muy alta (5).

\section{Resultados y discusión}

$\mathrm{E}^{\mathrm{n}}$ la Figura 3 se muestran los distintos mapas temáticos usados como indicadores para la obtención de la vulnerabilidad ambiental de la Cuenca Alta del río San Pedro (CARSP). La Figura 3a relacionado con el mapa del modelo digital de elevación (MDE), presentó un rango de elevación que osciló de 1022 a 1950 m. Las zonas de mayor intervención antrópica se localizan en las áreas de elevación media (1404$1586 \mathrm{~m})$ y baja (1040-1400 m) caracterizada por una intensa actividad agrícola, principalmente hortalizas y flores, con manejo que incluye preparación de suelos, alto uso de insumos agrícolas (fertilizantes, herbicidas, insecticidas), uso industrial que sumado a la deforestación y los incendios constituyen una de las principales amenazas de la zona (Castillo y Salas, 2006; Marín y Bravo, 2013). La deforestación y los incendios en esta zona, refleja una condición biótica que da paso a una etapa sucesional con una vegetación de sabana y confiere una mayor sensibilidad frente a los procesos morfodinámicos (Marín y Bravo, 2013).

Las zonas de mayor elevación se correspondieron con las áreas más boscosas localizadas dentro del Parque Nacional
Macarao. Como ha sido señalado el MDE, constituye una guía completa para el estudio de la morfología de una zona, por tanto, se pudo obtener las variaciones de pendiente y la red de drenaje de la mencionada cuenca, parámetros importantes que definen el nivel de escorrentía que puede fluir hacia un sector determinado.

La Figura $3 \mathrm{~b}$ muestra el mapa de pendientes de la CARSP obtenido a partir del MDE. Se puede apreciar que la subcuenca presenta una gran variación espacial de pendiente, oscilando valores muy suaves $(0-15 \%)$ a zonas de pendientes media a altas, incluso mayores de $60 \%$. La zona montañosa del área de estudio predomina pendientes que oscilaron entre 30 y $60 \%$, mientras, las áreas de menor pendiente se ubicaron en los márgenes del río San Pedro, que corresponde a la zona donde están asentadas la mayoría de las comunidades.

Si bien, se puede apreciar una gran variación de pendiente en la subcuenca, las zonas de mayor desarrollo de vivienda y actividad agrícola denominadas sector río Arriba, Andrés Bello y el Casco Central de la ciudad de San Pedro se caracterizan por presentar pendientes que van de 0 a $5 \%$, sugiriendo un mayor riesgo a crecidas e inundaciones. Sin embargo, los bordes montañosos o el resto de las comunidades, presentan rangos de pendientes mayores de $45 \%$. Los distintos rangos de pendientes son indicadores importantes para conocer el nivel de criticidad, al suponer que áreas con pendientes mayores a $40 \%$ son las más críticas para el desarrollo de cualquier actividad antrópica (Primelles et al., 2004).

El mapa de uso de la tierra para la subcuenca alta del río San Pedro, muestra cinco tipos de usos (Figura 3c). El bosque alto denso con un área de 1909.58 ha representa el $61.92 \%$; bosque bajo con 538 ha (17.47\%); vegetación baja/ especies arbustivas 298.97 ha (9.70\%); construcciones 229.13 

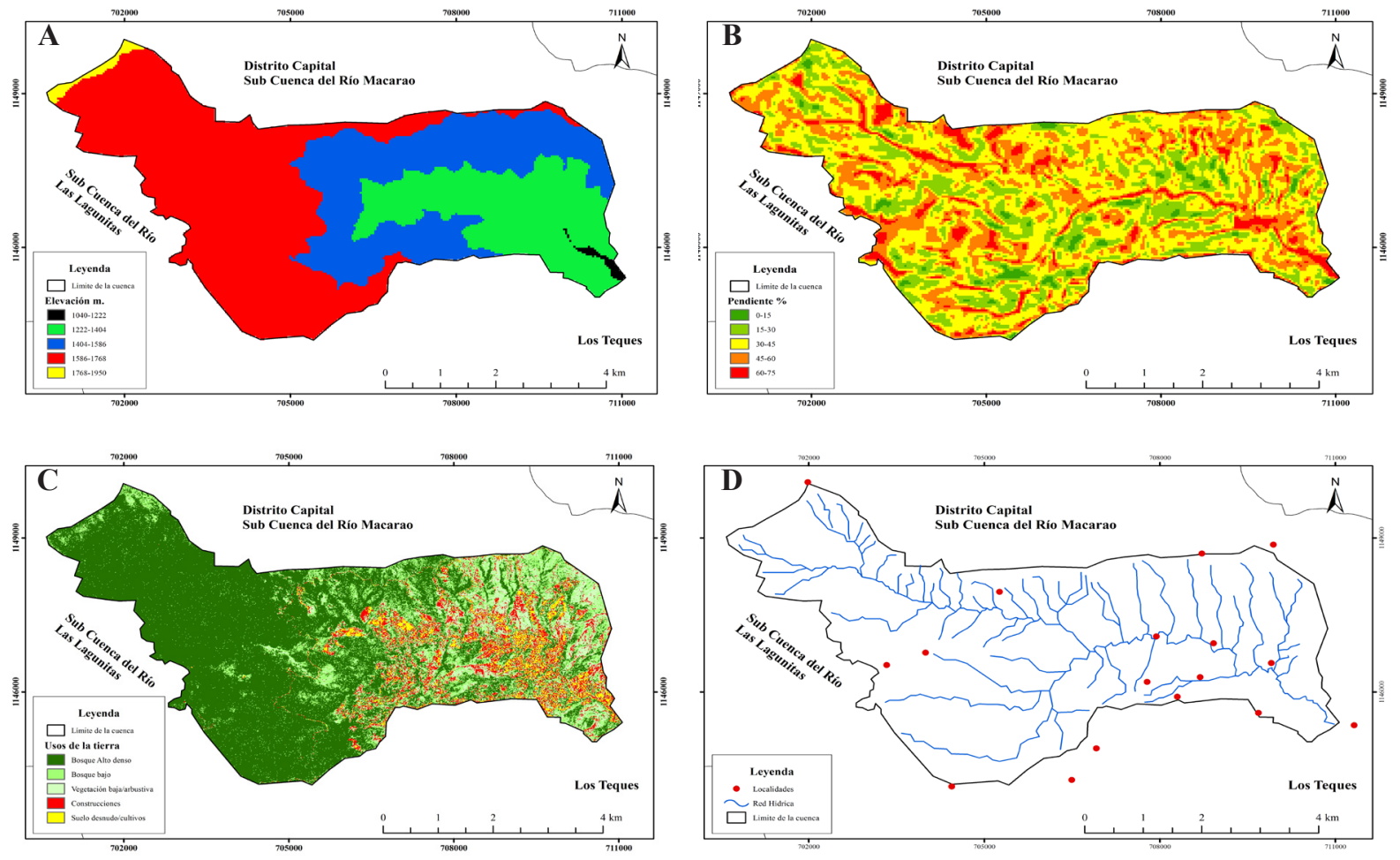

Figura 3. Mapas temáticos de la Cuenca Alta del río San Pedro, A) Modelo digital de elevación, B) Pendiente; C) Cobertura y D) red hidrológica

ha $(7.43 \%)$, y suelo desnudo/cultivos hortícolas 107.24 ha $(3.48 \%)$. En la zona bajo estudio las áreas de bosques representaron el $80 \%$ del área total de la subcuenca, mientras, el resto aproximadamente un $20 \%$ se distribuye en actividades relacionadas con la intervención antrópica, que se localiza en la zona señalada como B. Este comportamiento está vinculado, porque la zona se ubica en el parque nacional Macarao y en la parte menos intervenida predomina una área de bosque denso con algunos agregados de bosque bajo de aproximadamente de 2000 ha de superficie. Esta situación sirve de protección a la Cuenca y es la zona donde nace el rio San Pedro, por tanto, representa un gran reservorio de fauna y vegetación (Castillo y Salas, 2006).

Como se señaló anteriormente, el ámbito de estudio se dividió en dos zonas, una zona A no-intervenida con un área aproximada de 1733 ha $(56.2 \%$ del total de la subcuenca en estudio) que va desde el topo el arado hasta los límites de la quebrada Maturín y altos de la culebra y otra zona B fuertemente intervenida con una extensión 1347 ha (43.8\% del total de la Cuenca), caracterizada por una alta intervención antrópica. La actividad morfodinámica en la zona A (movimiento de materiales en forma de derrumbes, deslizamientos) tiene poco impacto debido a la existencia de áreas boscosas que atenúan el efecto de los agentes erosivos como la precipitación (Marín y Bravo 2013). Cuando se detalla la zona B se observa algunas zonas de bosque menos denso y vegetación baja asociada principalmente a los bordes del río principal (río San Pedro), a las quebradas o riachuelos y a los drenajes naturales. No obstante, en esta zona sobre todo cercana a los márgenes del río y en las zonas de ladera es donde se asienta la mayor intervención antrópica, bien sea por las construcciones de galpones o viviendas familiares o áreas dedicadas a la actividad agrícola. Caracterizada principalmente por la siembra de hortalizas como coliflor (Brassica oleracea, var Botrytis), Brócolis (Brassica oleracea, var Itálica), berro (Nasturtium officinale), Cebollín (Allium schoenoprasum), cilantro (Coriandrum sativum), acelga (Beta vulgaris subsp. Vulgaris), repollo (Brassica oleracea var. Capitata), apio españa (Apium graveolens), lechuga (Lactuca sativa) y flores ornamentales como: crisantemos (Chrysanthemum morifolium), gerbera (Gerbera jamesonii), margarita (Bellis perennis), rosas (Hibiscus syriacus), girasoles (Helianthus $\mathrm{sp})$, dalias (Dahlia spp), ave del paraíso (Strelitzia reginae), narcisos (Narcissus tazetta), solidaster (Aster alpinus). Ambos manejados con un enfoque convencional que incluye distintas prácticas agrícolas como preparación de suelos y alto uso de insumos agrícolas (fertilizantes, herbicidas, insecticidas). Igualmente, para el avance de la frontera agrícola se ha venido deforestando y quemando, lo cual se refleja en una condición biótica que da lugar al desarrollo de una vegetación de sabana, confiriéndole a estos lugares mayor sensibilidad frente a los procesos morfodinámicos. Los pequeños productores son quienes principalmente se dedican a estos cultivos en la parte de ladera y baja de la subcuenca. 
En cuanto a la red de hidrológica (Figura 3d) la misma está conformada por el río San Pedro como principal curso de agua, el cual es de régimen permanente, de bajo caudal, caracterizado por un cauce bien definido, poco profundo, taludes inclinados, y ancho variable, entre 3 a 7 metros (Urbina, 2009; Marín y Bravo, 2013). Por otra parte, la zona cuenta con gran cantidad de acuíferos (terreno con agua) que se recargan de los escurrimientos montañosos donde se producen altas precipitaciones que se infiltran entre las rocas fraccionadas y también escurren hacia las diferentes quebradas que alimentan otros acuíferos (Urbina, 2009).

La extracción de las características hidrológicas a partir de un Modelo de Elevación Digital- MED muestra un conjunto de parámetros morfométricos y de capacidad hidráulica, que le confieren altas posibilidades de ocurrencia de desbordes (Marín y Bravo, 2013). Como ha sido señalado, la forma de la cuenca determina la distribución de las descargas de agua a lo largo del curso principal y es en gran parte el responsable de las características de las crecientes que se pueden presentar (Santillán et al., 2012). El factor de forma con un valor de 0.25 y el índice de alargamiento (2.68), muestran una cuenca de forma alargada y está menos sujeta en términos generales a las crecidas. También las características físicas de la cuenca y la mayor parte del comportamiento hidrológico se encuentran influenciadas por la topografía, puesto que a mayores pendientes se corresponden con mayores velocidades de las corrientes de agua y menor será el tiempo de concentración. Por el contrario, cuando se produce cambios de pendiente de zonas muy inclinadas a zonas planas, aumenta el tiempo de concentración y las posibilidades de inundación, tal es el caso de algunas comunidades llamadas Rio Arriba, Andrés Bello y Casco Central.

\section{Vulnerabilidad por inundación y desplazamiento.}

La Figura 4a y 4b muestra los mapas de vulnerabilidad a inundación y desplazamiento, respectivamente. El grado de vulnerabilidad por inundación indica que los valores más altos se obtuvieron en las zonas más cercanas al cauce del rio en toda el área de la cuenca. La clase de vulnerabilidad media (3) fue la que representó el mayor porcentaje $(33.75 \%)$ de todo el territorio de la subcuenca, seguido por la clase 2 (baja) con un $31.91 \%$, por la clase 1 (muy baja) con un $26.86 \%$, clase 4 (alta) $6.12 \%$ y la clase 5 categorizada muy alta con sólo 1.37\% del territorio. Si bien, los menores porcentajes de vulnerabilidad se obtienen para las clases alta (4) y muy altas (5), dichas clases se corresponde con zonas donde se ubican la mayoría de las comunidades, caracterizadas por la presencia de desarrollos urbanos, alta actividad agrícola, lo cual le confiere un alto riesgo por inundación con sus correspondientes daños materiales y humanos. Las inundaciones se producen por desborde de ríos y lagos, que cubren temporalmente los terrenos bajos adyacentes a las riberas. La Parroquia San Pedro está conformado por más de 50 sectores, organizados en 32 Consejos Comunales, de las cuales 20 (40\% del total) se encuentran localizadas dentro del área de la subcuenca alta del Río San Pedro. La población de la cuenca es aproximadamente de 9175 habitantes, que representan el $70.50 \%$ del total de la parroquia, lo que determina el comportamiento del patrón poblacional dentro del área objeto de estudio. La ubicación de un alto porcentaje de comunidades en la Zona B, matiza la localización y características de la infraestructura social del territorio, que lógicamente es más densa y mejor provista en la zona, aunque insuficiente, si se tiene en cuenta la presión poblacional que recibe (Marín y Bravo, 2013). En especial, se perciben como altamente vulnerables a las comunidades Río Arriba, Casco Central, San Rafael, Ventorrillo, Andrés Bello y el Topo. Si bien, se obtiene esa distribución espacial de la vulnerabilidad, al realizar la superposición del mapa de las comunidades con el mapa de vulnerabilidad a inundación, a pesar de que los menores porcentajes de vulnerabilidad se obtienen para las clases alta (4) y muy altas (5), se aprecia que dichas clases se corresponden con zonas donde se ubican la mayoría de las comunidades, caracterizadas por la presencia de desarrollos urbanos, alta actividad agrícola, lo cual le confiere un alto riesgo por inundación con sus correspondientes daños materiales y humanos.

\section{Vulnerabilidad por deslizamiento.}

La distribución espacial de la vulnerabilidad por desplazamiento de la subcuenca alta del río San Pedro (Figura $4 b)$, mostró una predominancia de las clases de vulnerabilidad media (3) y baja (2), las cuales representan el 50.58 y $36.25 \%$, respectivamente. No obstante, las clases alta (4), muy baja (1) y muy alta (5) mostraron porcentajes que oscilaron de 9.37 , 3.29 y $0.41 \%$ respectivamente.

La estructura geológica y tipos de suelos de la zona permiten inferir el grado de estabilidad de un área en particular. Se ha señalado que el área de estudio se corresponde con dos formaciones geomorfológicas (Las Brisas y Las Mercedes) que se superponen una sobre otra y cuyo material litológico está compuesto de esquistos calcáreos, feldespato, caliza metaconglomerado, cuarcitas, zonas arenosas, grafitosas y localmente micáceas (Veitia y Malapina, 1994). La superposición de las formaciones produce unas fallas de corrimiento lo que aumenta los riesgos de inestabilidad derivados de los movimientos del suelo y subsuelo. Igualmente, la mayoría de estas laderas se encuentran en condiciones de desequilibrio potencial; observándose una concentración de los movimientos de masa en las zonas de cabecera, donde la saturación es mayor; un desmejoramiento del comportamiento mecánico de la parte superficial más arcillosa de los perfiles de meteorización ligado a la erradicación de la cobertura boscosa en equilibrio con ellos, y bajo el efecto de ciclos de desecación mucho más frecuentes y profundos que antes. Estos ciclos afectarían sobre todo las arcillas illíticas caracterizadas por coeficientes de retracción más elevados y concentradas en las laderas. Se iniciaría de esta manera un proceso de degradación geomorfológica de los terrenos acompañado de la aparición 

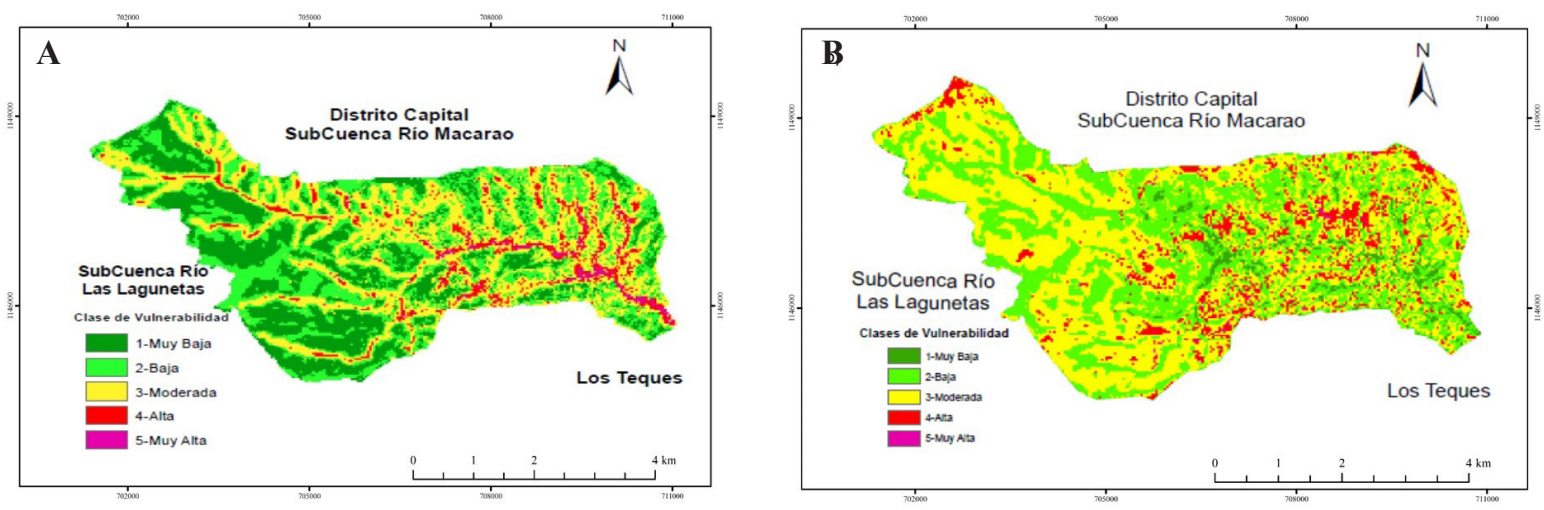

Figura 4. Clases de Vulnerabilidad por inundación (A) y desplazamiento (B) de la subcuenca Alta del Rio San Pedro, Miranda, Venezuela

de grietas de "suffosión" favoreciendo la saturación local de los materiales y el incremento de los movimientos de masa se combina con una concentración de la escorrentía superficial a partir de la conversión de las grietas de suffosion en zanjas de erosión e incluso en cárcavas (Veitia y Malapina, 1994).

Cuando la vulnerabilidad se analiza por zonas, se aprecia que las clases de vulnerabilidad alta y muy alta se corresponden con zona de mayor intervención (Zona B), con un patrón muy irregular pero asociado a suelos con altas pendientes y usos de la tierra con cultivos pocos protectores como las hortalizas, donde la preparación de tierra deja el suelo desnudo, desprovisto de vegetación favoreciendo procesos de degradación, principalmente erosión hídrica (Bravo y Florentino, 1999; Pla, 2010b).

Las características topográficas del terreno como la pendiente y longitud de la cuenca (Figura 3c) influyen marcadamente la velocidad de la escorrentía superficial y afecta el tiempo que tarda la lluvia para concentrarse en los lechos fluviales, que constituyen la red de drenaje de la cuenca (Pla, 2010b). En base a ello, la velocidad de escurrimiento de las corrientes de agua es directamente proporcional a la pendiente. Pendientes marcadas, como las que se presentan en la subcuenca alta, favorecen la escorrentía superficial, mientras que bajos valores, menores a 5\%, como algunas zonas de la cuenca, favorecen la infiltración o zonas de encharcamiento. Por tanto, la subcuenca presenta riesgo de desplazamiento de las capas del suelo o deslizamientos de tierras por la marcada pendiente (Ruíz, 2001).

Sumado a las características topográficas, el mal manejo de los recursos suelo y agua puede conducir a una acelerada degradación. La degradación de suelos entendida como un descenso en la habilidad del suelo para cumplir con algunas de sus funciones, entre ellas medio para el crecimiento de las plantas, como regulador del régimen hídrico y como filtro ambiental (McBratney et al., 2014). Como efectos indirectos de la degradación de suelos y agua, se presentan riesgos crecientes de desastres naturales (inundaciones, sedimentaciones, deslizamientos de tierra, etc.), así como disminución de la biodiversidad, deterioro de la suplencia de agua en cantidad y calidad, y efectos en cambios climáticos globales y sus consecuencias con características a veces catastróficas (Pla, 2010a).

Las actividades humanas pueden afectar, en forma más o menos determinante, la frecuencia y severidad de muchos de ellos. Entre ellas destacan el crecimiento de la población y de sus necesidades, la concentración de la población en grandes centros urbanos, y la localización de desarrollos urbanos e industriales en zonas vulnerables a desastres relacionados con el clima.

Al observar el mapa de uso de la tierra (Figura 3c) y contrastarlos con el mapa de las comunidades se puede señalar que la mayoría de las mismas son altamente vulnerables al problema de deslizamiento, fundamentalmente debido a las características topográficas, geológicas y de manejo de la zona (Urbina, 2009). Si bien, existe un alto porcentaje de la subcuenca bajo bosque, las áreas de mayor intervención como la zona B, con una intensa actividad agrícola junto a los distintos rangos de pendiente de la subcuenca, son indicadores importantes que contribuyen a esta vulnerabilidad, al observar que predominan pendientes entre 30 y $45 \%$ en la mayoría de la zona bajo estudio.

\section{Conclusiones}

$\mathrm{E}^{\mathrm{n}}$ n general, la vulnerabilidad por inundación varia de media a muy baja, sumando un $92 \%$ del territorio de la subcuenca, lo cual está asociado a la topografía. Si bien, las clases vulnerabilidad alta y muy alta representaron los menores porcentajes, el riesgo es mucho mayor debido a que corresponde con zonas donde se ubican la mayoría de las comunidades cercanas al río, caracterizadas por la presencia de desarrollos urbanos, alta actividad agrícola, en especial las comunidades Río Arriba, Casco Central, San Rafael, Ventorrillo, Andrés Bello y el Topo. La distribución 
espacial de la vulnerabilidad por desplazamiento sugiere que predomina la clase media (3) y baja (2), las cuales representan el 87\%, mientras, las clase alta (4), muy baja (1) y muy alta (5) mostraron porcentajes de $9.37,3.29$ y $0.41 \%$, respectivamente. Las clases de vulnerabilidad alta y muy alta están localizadas en el área de mayor intervención (Zona B), con un patrón muy irregular pero asociado a suelos con altas pendientes y manejos de suelo con cultivos pocos protectores como las hortalizas, flores, con enfoque convencional sin base agroecológica, favoreciendo los procesos de escorrentía superficial y degradación de los suelos (erosión).

\section{Bibliografía}

Altieri, MA. y Nicholls, CI. (2013). Agroecología y resiliencia al cambio climático principios y consideraciones metodológicas. Agroecología 8: 7-20.

Bravo, C., y Florentino, A. (1999). Nivel de cobertura, conservación de suelos y aguas bajo diferentes sistemas de labranza. Revista Facultad Agronomía 25:57-74.

Buch, M., y Turcios, M. (2003). Vulnerabilidad socioambiental. Aplicaciones para Guatemala. Serie de Documentos Técnicos, N 9.Instituto de Agricultura, Recursos Naturales y Ambiente. IARNA_URL. Guatemala. Pp.1-23.

Castillo, R. y Salas, V. (2006). Estado de Conservación del Parque Nacional Macarao. En: "BioParques: Asociación Civil para la Conservación de los Parques Nacionales". Programa de Observadores de Parques. Disponible en: (www.bioparques.org / www.parkswatch.org)

Chuvieco, E. (2008). Teledetección Ambiental. Tercera Edición. Editorial Ariel. Barcelona- España 592 p.

Díaz, K. (2002). Estudio de impacto ambiental. Metro de los Teques: Tramo las Adjunta los Teques. Revista Geografía de Venezuela 43(1): 77-96.

Esmail, BA. y Geneletti, D. (2017). Design and impact assessment of watershed investments: An approach based on ecosystem services and boundary work. Environmental Impact Assessment Review 62: 1-13.

González, N., Jiménez, F., Velásquez, S. y Camero, A. (2003). Áreas críticas y vulnerabilidad a desastres naturales en las subcuenca Molino Norte y San Francisco, Matagalpa, Nicaragua. Comunicación Técnica. CATIE, Costa Rica $11 \mathrm{pp}$.

IPCC. (2008). Cambio climático 2007: Informe de síntesis. Contribución de los Grupos de trabajo I, II y III al Cuarto Informe de evaluación del Grupo Intergubernamental de Expertos sobre el Cambio Climático [Equipo de redacción principal: Pachauri, RK. y Reisinger, A. (directores de la publicación)]. IPCC, Ginebra, Suiza, 104 págs. Disponible en: (http://www.ipcc.ch/assessment-report/)

IUCN. (2004). IUCN Red List of Threatened Species.
Disponible en Internet: http://www.iucnredlist.org

Lavell, A. (2003). La Gestión local del riesgo. Nociones y precisiones con respecto al concepto y la práctica. Programa Regional para la Gestión del Riesgo en América Latina. PNUD. 101 pág. Guatemala.

Marín, H., y Bravo, C. (2013). Caracterización biofísica y social de la subcuenca alta del río San Pedro, estado Miranda. Entreciencias (1): 73-96.

McBratney, A., Field, D., Koch, A. (2014). The dimensions of soil security. Geoderma, 213: 203-213.

Pla, I. (2010a). Medición y evaluación de propiedades físicas de los suelos: dificultades y errores más frecuentes. I. Propiedades Mecánicas. Suelos Ecuatoriales 40(2): 75-93

Pla, I. (2010b). Medición y evaluación de propiedades físicas de los suelos: dificultades y errores más frecuentes. II. Propiedades hidrológica. Suelos Ecuatoriales 40(2): 94127.

Primelles, F., Zequeira, M., Acosta, Z., Martín, Ferrer, G., Ferrer, A., Brito, O., Hernández, L., Machado, W., Aguilar, J., Plasencia, J. (2004). Cuenca del Río San Pedro: Diagnóstico Ambiental y Objetivos para su Manejo Integrado. Revista Electrónica de la Agencia de Medio Ambiente, Año 4, N 7. Disponible en (http://ama. redciencia.cu/articulos/7.06.pdf).

Ruiz, J. (2001). Hidrología, evolución y visión sistémica. La morfometría de cuencas como aplicación. Fondo Editorial UNELLEZ. Barinas-Venezuela. 298 p.

Santillán, E., Jarquin, Y., Anda, J. y Olvera, M. (2012). Análisis morfométrico y delimitación de cuencas hidrográficas de topografía compleja. Disponible en http://www. selper-mexico.org.mx/XT\%20PDF/HIDROLOGIA/ HIDRO-01.pdf

Smith, P. y Olesen, JE. (2010). Synergies between the mitigation of, and adaptation to, climate change in agriculture. Journal of Agricultural Science, 148, 543552.

Urbina, C. (2009). Disponibilidad de agua en la subcuenca alta del río san Pedro, estado Miranda. MPP para el Ambiente, Dirección General de Cuenca Hidrográficas, Caracas, Agosto, 33 pp.

Veitia, G., Malapina, H. (1994): "Diagnostico Geográfico de la Parroquia San Pedro, Estado Miranda, con fines de Ordenamiento Espacial", Trabajo Especial de Grado, titulación de Geógrafo. Universidad Central de Venezuela.

Wilchex-Chaux, G. (1993). La Vulnerabilidad Global. En: Maskrey A Editores. Los Desastres no son naturales. La RED Tercer Mundo Editores, Bogotá- Colombia p. 9-50.

Zevenbergen, L. and Thorne, C. (1987). Quantitative analysis of land surface topography. Earth Surface Processes and Landform 12(1), 47-56. 